\title{
Semantic methods in genetic programming
}

\author{
Michael O'Neill ${ }^{1}$
}

Published online: 30 October 2015

(C) Springer Science+Business Media New York 2015

We are delighted to present this special issue of Genetic Programming and Evolvable Machines on Semantic Methods in Genetic Programming. Genetic programming (GP) - the application of evolutionary computing techniques to the creation of computer programs - has been a key topic in computational intelligence in the last couple of decades. In recent years an emerging topic in GP has been the use of semantic methods. The aim of this Special Issue is to provide a way of exploring the input-output behaviour of programs, which is ultimately what matters for problem solving. This contrasts with much previous work in GP, which has a syntactical focus, where operators transform the program text and the effect on program behaviour is indirect. The use of semantic methods in GP has substantially improved the performance of GP on a number of problems, including both benchmarks and real-world applications. Semantic methods in GP have also been grounded in a body of theory, which helps to inform algorithm design.

Following an open call for papers, this issue is comprised of three articles with a common thread of geometric semantic operators, a very popular and fruitful direction of research within the community, which exploit geometric properties of program semantics.

The special issue was preceded by two international workshops on Semantic Methods in Genetic Programming, the first held at PPSN (13-17 September 2014 in Ljubljana, Slovenia) the 13th International Conference on Parallel Problem Solving from Nature, and the second at the ACM Genetic and Evolutionary Computation Conference GECCO 2015 (11-15 July 2015 in Madrid, Spain). The three articles

Michael O’Neill

m.oneill@ucd.ie

1 Natural Computing Research and Applications Group, School of Business, University College Dublin, Dublin, Ireland 
have undergone two rounds of rigorous peer-review, and we would like to thank the reviewers involved for their invaluable input.

The first article, by Tomasz P. Pawlak and Krzysztof Krawiec on "Progress Properties and Fitness Bounds for Geometric Semantic Search Operators" undertakes a theoretical analysis on the role of metrics for geometric semantic approaches to genetic programming.

The second article on "Subtree Semantic Geometric Crossover for Genetic Programming" is by Quang Uy Nguyen, Tuan Anh Pham, Nguyen Xuan Hoai and James McDermott, and presents an approach to address the exponential growth in the size of solutions observed using standard geometric semantic genetic programming.

The third article is by Mauro Castelli, Luca Manzoni, Leonardo Vanneschi, Sara Silva and Aleš Popovič on "Self-Tuning Geometric Semantic Genetic Programming" presents an approach to self-tune the probabilities of mutation and crossover.

While the three articles in this special issue are important contributions to our community, and represent a current hot topic in Genetic Programming research, we shouldn't lose sight of the fact that as it stands the geometric semantic approaches to genetic programming are not yet capable of general program evolution, and in their canonical form are difficult to use in practice due to the code growth problem. It will be interesting to see if this new emphasis on the use of semantics for Genetic Programming will extend beyond its current focus on search operators towards some initial seeds of research on semantic-aware program construction, and semantic analysis of evolving populations for, for example, credit assignment and automatic identification of useful semantic modules.

We hope you enjoy this special issue and take some inspiration from it for your own future research. 